\title{
Revenue Sharing Coordination Mechanism of Supply Chain under Disruptions
}

\author{
Weiwei Ji*, Baohua Zhang, Hengmin Lv \\ Department of Basic Course Education, Ji'an College, Ji'an, China \\ Email: *jiweiwei0828@126.com
}

How to cite this paper: Ji, W.W., Zhang, B.H. and Lv, H.M. (2019) Revenue Sharing Coordination Mechanism of Supply Chain under Disruptions. Open Journal of Social Sciences, 7, 32-37. https://doi.org/10.4236/jss.2019.710003

Received: September 17, 2019

Accepted: October 7, 2019

Published: October 10, 2019

Copyright $\odot 2019$ by author(s) and Scientific Research Publishing Inc. This work is licensed under the Creative Commons Attribution International License (CC BY 4.0).

http://creativecommons.org/licenses/by/4.0/

\section{(c) (i) Open Access}

\begin{abstract}
This paper studies the coordination problem of a supply chain which is consisted of one supplier and one retailer. Based on a mathematical model, this paper analyzes how to adjust the production plan and pricing strategy to maximize the profit of the supply chain when market scale, price sensitivity coefficient and product cost are disrupted. We assume that the supplier and the retailer adopt revenue-sharing contract to coordinate the supply chain. If the mutation is small, the decision-maker wouldn't adjust the production plan. If the mutation is large, the decision-maker had better adopt an improved revenue-sharing contract which has a stronger anti-disruption ability to adjust the production plan and pricing strategy. The new contract can coordinate the supply chain under mutation and realize the optimal allocation of the supply chain's profit.
\end{abstract}

\section{Keywords}

Supply Chain Coordination, Disruptions, Revenue-Sharing Contract

\section{Introduction}

As the global economy develops fast, thousands of global enterprises have benefited from a new subject named supply chain management. But in reality, supply chain system often encounters various emergencies because of unsteady external environment and inaccurate forecast information. Sudden natural disasters would cause different levels of interference to the supply chain. Such as pig epidemic in 2019, it has a great impact on meat processing companies and affects consumers' demand for pork. Lanzhou water pollution in 2014 leaded to the increase of mineral water price and short supply of the market demand. Earthquakes, epidemic, terrorist attacks, these emergencies all have a great influence on the normal operations of the system to some extent, and some would cause disruptions 
in the supply chain. So how to coordinate the supply chain to deal with emergencies more quickly and effectively becomes particularly important.

Many scholars make intensive researches on supply chain disruption management. Li et al. [1] examined the problem of redesigning revenue sharing contract parameters under cost and demand disruption with linear and exponential demand functions. Introducing promotional service costs, Chen et al. [2] studied the adjustment of the optimal wholesale price, order quantity and subsidy rate when the demand changes. Liu [3] proposed an option contract mechanism that can make the supply chain coordination when the retailer is in leading position. Rhee [4] proposed three different forms of supply chain contact mechanism to make the supply chain reach coordination in the case of suppliers and retailers with capacity limitations. Bozorgi A [5] used a buyback contract to coordinate the supply chain with stochastic demand when both the cost and price were disrupted.

Most of the literature mentioned above focus on one or two factors, but investigations on three factors' disruption are less. In this paper, we'll establish a game model on three factors' disruption. The supplier is the leader while the retailer is follower. Supplier and retailer are both risk-neutral and rational, independent decision-makers. They both focus on their own interests respectively. Information such as retail price of products and inventory cost is symmetric.

\section{Basic Model without Disruption}

In order to facilitate the theoretical model, we assume that the product is seasonal, production and sales occur in one cycle, we have the following notions:

The market demand function is $d=D \mathrm{e}^{-\alpha p}, D$ represents the market scale, $\alpha$ represents the price sensitive coefficient, $p$ represents the retail price respectively. In normal situations, the retailer's profit $R_{r}(Q)=Q\left(\frac{1}{\alpha} \ln \frac{D}{Q}-w-c_{r}\right)$, the supplier's profit $R_{s}(Q)=Q\left(w-c_{s}\right)$, the supply chain's profit $R_{s c}(Q)=Q\left(\frac{1}{\alpha} \ln \frac{D}{Q}-c\right)$, where $c=c_{r}+c_{s}, \quad c_{s}$ represents the unit production cost, $c_{r}$ represents the unit retail cost and $w$ represents the unit wholesale price.

Then we can obtain the first-order derivatives of $R_{s c}(Q)$ with respect to $Q$, $\frac{\partial R_{s c}(Q)}{\partial Q}=0$.

We can get $\bar{Q}=D \mathrm{e}^{-(1+\alpha c)}, \bar{P}=\frac{1}{\alpha}+c$, the maximum supply chain's profit $R_{s c}(\bar{Q})=\frac{D \mathrm{e}^{-(1+\alpha c)}}{\alpha}$.

According to the conclusions drawn by Cachon in research [6] we know if $w=\theta c-c_{r}$, where $0<\theta<1$, the supply chain is coordinated. $\theta$ is the retailer's share of the supply chain's profit. We call this coordination mechanism the 
original strategy.

\section{Centralized Decision-Making with Demand Disruption}

Supply chain works as a system under the case of centralized decision-making, in order to maximize the profit. The parameters $c$ and $p$ are decision variables. We determine an optimal order quantity to achieve Pareto Optimality. $\Delta D$, $\Delta c, \Delta \alpha$ represents the changed amount of market scale, product cost and price sensitive coefficient, respectively. Only if $\Delta D+D>0, \Delta c+c>0$, $\Delta \alpha+\alpha>0$ does the case have practical meaning. When emergencies occur, the supply chain's profit is:

$$
R_{s c}(Q)=Q\left(\frac{1}{\alpha+\Delta \alpha} \ln \frac{D+\Delta D}{Q}-(c+\Delta c)\right)-k_{1}(Q-\bar{Q})^{+}-k_{2}(\bar{Q}-Q)^{+}
$$

when $(x)^{+}=\max \{0, x\}, \quad k_{1}$ represents the unit penalty cost for one unit increment and $k_{2}$ represents the unit penalty cost for one unit increment paid by supplier. $(Q-\bar{Q})^{+}$and $(\bar{Q}-Q)^{+}$are mutually exclusive events.

We assume $Q^{*}$ is the optimal order quantity of (1).

Lemma 1. When $\Delta D \geq\left(\mathrm{e}^{\Delta c(\alpha+\Delta \alpha)}-1\right) D$, we have $Q^{*} \geq \bar{Q}$; When $\Delta D<\left(\mathrm{e}^{\Delta c(\alpha+\Delta \alpha)}-1\right) D$, we have $Q^{*}<\bar{Q}$.

\section{Proof of Lemma 1:}

We make an assumption when $\Delta D \geq\left(\mathrm{e}^{\Delta c(\alpha+\Delta \alpha)}-1\right) D$, we have $Q^{*}<\bar{Q}$.

$$
\begin{aligned}
F_{s c}\left(Q^{*}\right) & =Q^{*}\left(\frac{1}{\alpha+\Delta \alpha} \ln \frac{D+\Delta D}{Q^{*}}-(c+\Delta c)\right)-k_{2}\left(\bar{Q}-Q^{*}\right) \\
& =Q^{*}\left(\frac{1}{\alpha+\Delta \alpha} \ln \frac{D}{Q^{*}}-c\right)+Q^{*}\left(\frac{1}{\alpha+\Delta \alpha} \ln \frac{D+\Delta D}{D}-\Delta c\right)-k_{2}\left(\bar{Q}-Q^{*}\right) \\
& <\bar{Q}\left(\frac{1}{\alpha+\Delta \alpha} \ln \frac{D}{\bar{Q}}-c\right)+\bar{Q}\left(\frac{1}{\alpha+\Delta \alpha} \ln \frac{D+\Delta D}{D}-\Delta c\right)-k_{2}\left(\bar{Q}-Q^{*}\right) \\
& \leq \bar{Q}\left(\frac{1}{\alpha+\Delta \alpha} \ln \frac{D}{\bar{Q}}+\frac{1}{\alpha+\Delta \alpha} \ln \frac{D+\Delta D}{D}-(c+\Delta c)\right)=R_{s c}(\bar{Q})
\end{aligned}
$$

We can see that $Q^{*}$ makes the maximum $R_{s c}(Q)$, so the assumption we made is a false proposition, that is, When $\Delta D \geq\left(\mathrm{e}^{\Delta c(\alpha+\Delta \alpha)}-1\right) D$, we have $Q^{*} \geq \bar{Q}$. In the same way we can obtain that When $\Delta D<\left(\mathrm{e}^{\Delta c(\alpha+\Delta \alpha)}-1\right) D$, we have $Q^{*}<\bar{Q}$. So lemma 1 is proved.

Then we classify the disruption influence by $\Delta D$.

When $\Delta D \geq\left(\mathrm{e}^{\Delta c(\alpha+\Delta \alpha)}-1\right) D$, from lemma 1, we can know the supply chain's profit is:

$$
R_{s c}(Q)=Q\left(\frac{1}{\alpha+\Delta \alpha} \ln \frac{D+\Delta D}{Q}-(c+\Delta c)\right)-k_{1}(Q-\bar{Q})
$$

The optimal order quantity is the one that makes the maximum $R_{\mathrm{sc}}\left(Q^{*}\right)$.

Then we can obtain the first-order derivatives of $R_{s c}(Q)$ with respect to $Q$. 
$\frac{\partial R_{s c}(Q)}{\partial Q}=0$, We can get $Q_{1}=(D+\Delta D) \mathrm{e}^{-\left[1+\left(c+\Delta c+k_{1}\right)(\alpha+\Delta \alpha)\right]}$.

1) If $\Delta D \geq\left[\mathrm{e}^{k_{1}(\alpha+\Delta \alpha)+\Delta c(\alpha+\Delta \alpha)+c \Delta \alpha}-1\right] D$, there exists a quantity $Q_{1}$ among the interval $[\bar{Q},+\infty)$, that makes the maximum $R_{s c 1}(Q)$, and $Q^{*}=Q_{1}=(D+\Delta D) \mathrm{e}^{-\left[1+\left(c+\Delta c+k_{1}\right)(\alpha+\Delta \alpha)\right]}, \quad p^{*}=\frac{1}{\alpha+\Delta \alpha}+c+\Delta c+k_{1}$.

2) If $\left(\mathrm{e}^{\Delta c(\alpha+\Delta \alpha)}-1\right) D \leq \Delta D<\left[\mathrm{e}^{k_{1}(\alpha+\Delta \alpha)+\Delta c(\alpha+\Delta \alpha)+c \Delta \alpha}-1\right] D$, there isn't a quantity $Q$ among the interval $[\bar{Q},+\infty)$ that makes the maximum $R_{s c}\left(Q^{*}\right)$. When $Q^{*}=\bar{Q}=D \mathrm{e}^{-(1+\alpha c)}$, the optimal retail price $p^{*}=\frac{1}{\alpha+\Delta \alpha} \ln \left(\frac{D+\Delta D}{D}\right)+\frac{1+\alpha c}{\alpha+\Delta \alpha}$.

\section{Decentralized Decision-Making with Demand Disruption}

Supplier and retailer make decisions independently under the case of decentralized decision-making. Supplier should offer a suitable wholesale price to induce the retailer to order a proper quantity.

Lemma 2. After emergencies happens, supplier adopts a new revenue sharing contract, contract parameters combination is $(w, \theta)$, the new wholesale price $w=\theta\left(c+\Delta c+\frac{k_{1}(Q-\bar{Q})^{+}+k_{2}(\bar{Q}-Q)^{+}}{Q}\right)-c_{r}$. In this way we can make

$R_{r}=\theta R_{s c}$ and coordinate the supply chain. We call this coordination mechanism the new strategy.

\section{Proof of Lemma 2:}

$$
\begin{aligned}
R_{r} & =\theta Q\left(\frac{1}{\alpha+\Delta \alpha} \ln \frac{D+\Delta D}{Q}\right)-w Q-c_{r} Q \\
& =\theta Q\left(\frac{1}{\alpha+\Delta \alpha} \ln \frac{D+\Delta D}{Q}\right)-Q\left[\theta\left(c+\Delta c+\frac{k_{1}(Q-\bar{Q})^{+}+k_{2}(\bar{Q}-Q)^{+}}{Q}\right)-c_{r}\right]-c_{r} Q \\
& =\theta Q\left(\frac{1}{\alpha+\Delta \alpha} \ln \frac{D+\Delta D}{Q}\right)-\theta Q\left(c+\Delta c+\frac{k_{1}(Q-\bar{Q})^{+}+k_{2}(\bar{Q}-Q)^{+}}{Q}\right) \\
& =\theta Q\left(\frac{1}{\alpha+\Delta \alpha} \ln \frac{D+\Delta D}{Q}-(c+\Delta c)\right)-\theta\left[k_{1}(Q-\bar{Q})^{+}+k_{2}(\bar{Q}-Q)^{+}\right] \\
& =\theta R_{s c}
\end{aligned}
$$

The retailer's profit function is an affine transformation of the supply chain's for all $Q$, through the parameter $\theta(0<\theta<1)$, we can allocate the supply chain's total profit between supplier and retailer arbitrarily, so the supply chain is coordinated. If we leave out the unit retail cost $c_{r}, W$ can be written as $\theta\left(c+\Delta c+\frac{k_{1}(Q-\bar{Q})^{+}+k_{2}(\bar{Q}-Q)^{+}}{Q}\right)$. Compared with the wholesale price under decentralized decision-making, we can see that the price sensitive coefficient 
disruption has nothing to do with the adjustment of optimal wholesale price.

\section{Numerical Examples}

We assume the original market scale $D=100$, supplier's unit production cost $c_{s}=4$, retailer's unit retail cost $c_{r}=1$, price sensitive coefficient $\alpha=0.2$. According to the first part of this paper we know $Q^{*}=13.5, P^{*}=10$, supply chain's total profit is 67.5. We suppose that in the supply chain, the supplier is the leader who is in a powerful position, having $60 \%$ profit of the supply chain, then the retailer's profit is $40 \%$, so $\theta=0.4, w=1$. The supplier's and retailer's profits are 40.5 and 27.

If the decision makers don't realize the influence of emergencies on supply chain, fails to adjust retail price timely and continues to use the original strategy, we can get the profit of original strategy. In the similar way, we can get the profit of new strategy. Results are analyzed to indicate the difference, which is as follows. The effect of the three factors on the system is shown in Table 1.

The examples shown above prove that when emergencies occur, the supplier can offer a proper $w$ to the retailer. We can see from Table 1 that system makes more profit with new strategy than original one, which means new contract indeed has great advantages over the original one.

\section{Conclusions}

This paper establishes models to investigate the effect of market scale, production cost and price-sensitive coefficient disruption on the supply chain system robustness with exponential demand function. We design a combination of appropriate contract parameters to make the optimal decisions under a decentralized decision-making situation to achieve the centralized level. We classify the influence caused by emergencies into safety range and dangerous range according to the market scale. The safety range is an interval in which the optimal decisions are free from disruption, the retailer needn't change order strategies because the system itself can resist the risks; the dangerous range is an interval in which the retailer has to adjust both pricing strategies and order strategies. The improved revenue-sharing contract has greater robustness which can coordinate the supply chain better after disruption and can also realize the optimal allocation

Table 1. Comparison of profit and wholesale price under different parameters.

\begin{tabular}{|c|c|c|c|c|c|c|}
\hline & \multirow{2}{*}{$\begin{array}{c}\text { Demand } \\
\Delta D\end{array}$} & \multirow{2}{*}{$\begin{array}{c}\begin{array}{c}\text { Sensitive } \\
\text { coefficient }\end{array} \\
\Delta \alpha\end{array}$} & \multirow{2}{*}{$\begin{array}{l}\text { Cost } \\
\Delta c\end{array}$} & \multicolumn{2}{|c|}{ Supply chain's profit } & \multirow{2}{*}{$w$} \\
\hline & & & & $\begin{array}{c}\text { Profit of original } \\
\text { strategy }\end{array}$ & $\begin{array}{c}\text { Profit of new } \\
\text { strategy }\end{array}$ & \\
\hline Case 1 & 60 & 0.02 & -0.02 & 84.56 & 85.00 & 0.99 \\
\hline Case 2 & 30 & -0.02 & 0.02 & 78.23 & 103.76 & 1.07 \\
\hline Case 3 & -10 & 0.02 & -0.1 & 46.32 & 61.18 & 0.96 \\
\hline Case 4 & -40 & -0.02 & 0.1 & 44.9 & 45.8 & 1.16 \\
\hline
\end{tabular}


of the supply chain's total profit. The numerical examples prove that the improved contract has considerable advantages.

This article also has many shortcomings. This article only studied a supply chain which is consisted of one supplier and one retailer, while in reality, the supply chain is complex. And the application research combined with the actual enterprise is the focus of future research in this paper.

\section{Conflicts of Interest}

The authors declare no conflicts of interest regarding the publication of this paper.

\section{References}

[1] Li, J., Wang, S. and Cheng, T.C.E. (2010) Competition and Cooperation in a Single-Retailer Two-Supplier Supply Chain with Supply Disruption. International Journal of Production Economics, 124, 137-150. https://doi.org/10.1016/j.ijpe.2009.10.017

[2] Chen, K. and Zhang, P. (2011) Disruption Management for a Dominant Retailer with Constant Demand-Stimulating Service Cost. Computer \& Industrial Engineering, 61, 936-946. https://doi.org/10.1016/j.cie.2011.06.006

[3] Rhee, B.D. (2017) Channel Coordination Using Product Returns for a Supply Chain with Stochastic Salvage Capacity. European Journal of Operational Research, 177, 214-238. https://doi.org/10.1016/j.ejor.2005.10.044

[4] Liu, L. (2016) Coordination in a Retailer-Led Supply Chain through Option Contract. International Journal of Production Economics, 127, 204-208.

[5] Bozorgi, A., Pazour, J. and Nazzal, D. (2014) A New Inventory Model for Cold Items that Considers Costs and Emissions. International Journal of Production Economics, 155, 114-125. https://doi.org/10.1016/j.ijpe.2014.01.006

[6] Cachon, G.P. (2005) Supply Chain Coordination with Revenue-Sharing Contracts: strengths and limitations. Management Science, 51, 30-44.

https://doi.org/10.1287/mnsc.1040.0215 